\title{
Arrayed Waveguide Grating Based on Group-Index Modification
}

\author{
Óscar M. Matos, María L. Calvo, Pavel Cheben, Siegfried Janz, Member, IEEE, \\ José A. Rodrigo, Dan-Xia Xu, and André Delâge
}

\begin{abstract}
The authors propose a new wavelength-dispersive principle based on waveguide group-index modification and apply this principle in a new type of arrayed waveguide dispersive element based on modified group index. The element is composed by an array of waveguides consisting of two sections with different group indexes. We deduce the applicable dispersion formula and demonstrate that the group-index modification can be used for controlling or enhancing device wavelength dispersion. Two device examples are provided. First, dispersive properties of a waveguide array with silicon on insulator (SOI) straight waveguides with group index modified by waveguide widening are calculated. Then, the authors show that by placing the element with modified group index in a phased array of a conventional arrayed waveguide grating (AWG) device, the dispersive properties of the AWG are markedly enhanced. Dispersion-enhancement factor of up to 60 is calculated for a compact demultiplexer designed for SOI platform with group index modified by photonic-bandgap effect.
\end{abstract}

Index Terms-Arrayed waveguide gratings (AWGs), silicon on insulator (SOI) technology, waveguide arrays, wavelength division multiplexing.

\section{INTRODUCTION}

T HE CONTINUOUS demand for Internet and telecommunication services is expected to keep driving the development of wavelength division multiplexed (WDM) networks [1], [2]. Wavelength multiplexers and demultiplexers, capable of combining and separating different spectral channels, are the key components of WDM network. The planar waveguidebased mux/demux devices include arrayed waveguide gratings (AWG) [3] and echelle grating devices [4]-[6]. State-of-the-art silica-on-silicon AWGs become prohibitively large for devices with higher channel counts and narrower channel spacing (CS) [7], and the integration of different functions on a single chip is not feasible for practical systems unless the size of the individual functional elements is significantly reduced. Silicon-

Manuscript received May 6, 2005; revised October 11, 2005. This work was supported by the Spanish Ministry of Science and Technology under project TIC2002-1846 and the National Research Council of Canada. Preliminary partial results were presented at the V Ibero-American Optics Meeting (RIAO) and VIII OPTILAS, October 2-8, 2004, Porlamar, Venezuela.

Ó. M. Matos, M. L. Calvo, and J. A. Rodrigo are with the Departamento de Óptica, Facultad de Ciencias Físicas, Universidad Complutense de Madrid, Madrid 28040, Spain (e-mail: omartine@fis.ucm.es; mlcalvo@ fis.ucm.es; jarmar@ fis.ucm.es).

P. Cheben, S. Janz, D.-X. Xu, and A. Delâge are with the Institute for Microstructural Sciences, National Research Council of Canada, Ottawa, ON K1A 0R6, Canada (e-mail: Pavel.Cheben@nrc-cnrc.gc.ca; Siegfried.Janz@ nrc-cnrc.gc.ca; Danxia.Xu@nrc-cnrc.gc.ca; Andre.Delage@nrc-cnrc.gc.ca).

Digital Object Identifier 10.1109/JLT.2005.863303 based photonic waveguide circuits have recently emerged as commercially viable optoelectronic devices [8]-[10]. In siliconon-insulator (SOI) waveguide devices, several orders of magnitude reduction of device size can be achieved [11], [12] as compared to devices based on silica-on-silicon materials. This is possible because of a very high difference in refractive index between the waveguide core ( $\mathrm{Si}, n \sim 3.5$ ) and the surrounding cladding material (typically $\mathrm{SiO}_{2}, n \sim 1.5$ ). AWG demultiplexers in SOI platform have been demonstrated [8], [11], [13], [14]. Ultracompact AWG devices using silicon wire waveguides have recently been reported [15], [16], but practical application of similar devices will require substantial improvements in quality of silicon waveguides of submicrometer cross-sectional dimensions, particularly the sidewall roughness.

Since conventional AWG demultiplexers require curved waveguides, the minimum available bend radius, which in turn is determined by the index contrast, sets the lower limit for the device dimensions. At the same time, AWG dispersion, and hence minimum achievable CS, is limited by a maximum available length difference between the waveguides in the phased array. Here, we propose a new dispersive element comprising the straight waveguides with sections of modified group index that, if placed in the phase array of a conventional AWG, can enhance dispersion properties of the latter. In the examples shown here, the group index is modified by changing the waveguide width or by the photonic-bandgap effect. Recently, Kawakita et al. [17] also proposed a straight waveguide AWG in which the refractive index is constant for each individual waveguide while it linearly increases from the first to the last waveguide in the phase array. The latter is achieved by the selected area growth of the waveguide layers, resulting in a changing waveguide thickness across the phase array. Fabrication challenges of such approach are obvious. These difficulties are obviated in our element because waveguide widths can be simply modified by lithography and no additional fabrication steps are required.

The proposed dispersive element does not necessary require curved waveguides, hence potentially yielding low loss devices compact in one (transversal) direction. In practical applications, imaging of the input waveguide on the output focal line is required. This can be achieved, for example, using a conventional AWG, a zero-order AWG geometry [see Fig. 1(a)] with relaxed requirements on waveguide bending compared to a conventional AWG, or avoiding the bends using waveguide lenses or mirrors. In the example described here, a conventional AWG geometry is used for imaging and combined with our element 


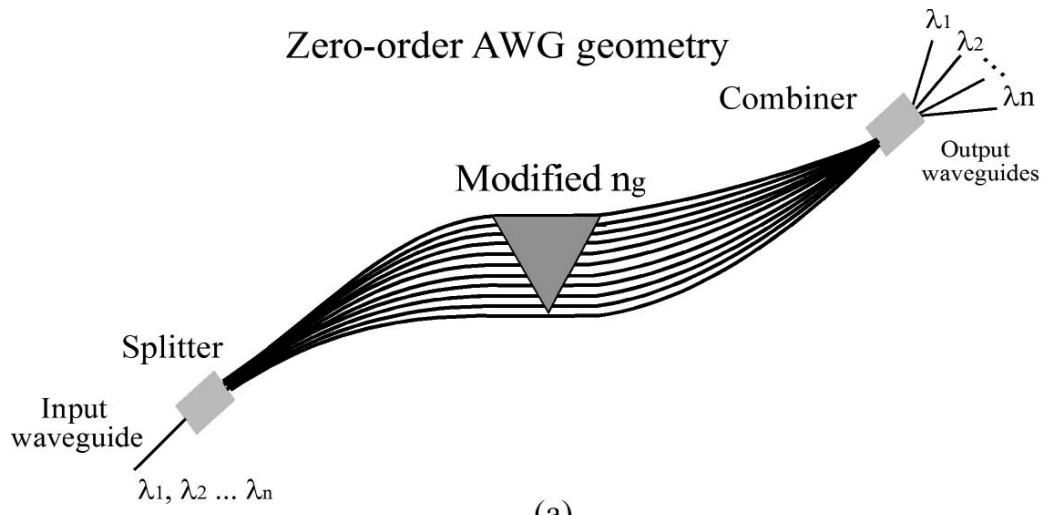

(a)

Dispersive Element

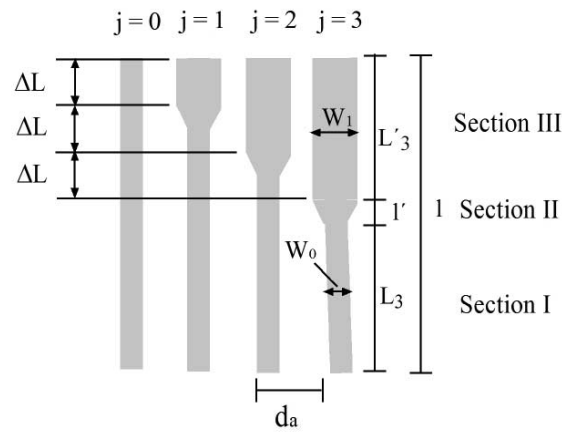

(b)

\section{Receiver geometry}

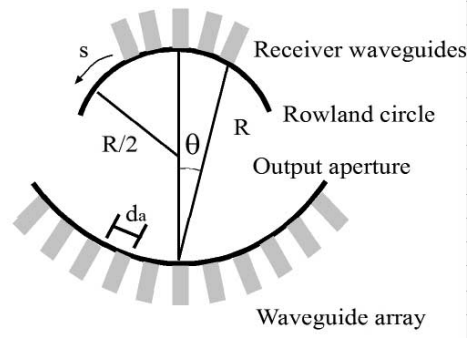

(c)

Fig. 1. (a) General schematics of a demultiplexing device implementing the dispersive element in a zero order AWG geometry. (b) Schematics showing four adjacent waveguides of the dispersive element. (c) Receiver geometry.

to enhance AWG dispersion. Another example of utilization of the proposed dispersive element is for interleaving two AWGs in transmission geometry of the recently suggested Fourier transform (FT) Michelson-type AWG spectrometer. It has been shown [18] that such FT AWG device has a markedly increased light throughput (étendue) that is relevant in spectroscopic applications. By interleaving two dispersive elements with different interference orders in a zero order AWG configuration, the FT AWG device can be made in transmission geometry that obviates the need for the relay optics and eliminates vignetting of a reflective configuration, as explained in [18].

\section{Dispersive Property OF THE WAVEguide ARRAY}

Fig. 1(a) shows a general schematics of a demultiplexing device in zero order geometry. An incoming beam from the input waveguide is coupled through slab-waveguide combiner (splitter) into the waveguide array and propagates through each individual waveguide toward the output aperture. Waveguides with modified group index forming the dispersive element are shown in Fig. 1(b). The element can also be placed in the central section of a conventional AWG device, as shown in Fig. 2.

First, we calculate the dispersive-element properties for a simple case of modified group index by changing the waveguide width. Then, we demonstrate dispersion enhancement in a conventional AWG device by placing sections of waveguides with a large group index in the phased waveguide array.
In the dispersive element comprising the waveguides with modified widths, each waveguide $j$ is divided into three different sections [see Fig. 1(b)]. The first section has a waveguide width $W_{0}$ and length $L_{j}$; the second section is a waveguide taper of length $l^{\prime}$; and the third section has a modified waveguide width $W_{1}$ and length $L_{j}^{\prime}$. We assume that the respective widths of the Sections I and III are constant, while their respective lengths change linearly between the adjacent waveguides and define the length increment $\Delta L\left(\Delta L^{\prime}\right)$ of Section I (Section III) between the adjacent waveguides $j$ and $j-1$ as $\Delta L=L_{j}-L_{j-1}$ and $\Delta L^{\prime}=L_{j}^{\prime}-L_{j-1}^{\prime} . \Delta L$ is constant for all the waveguides, and $\Delta L^{\prime}=-\Delta L$. The total length $l$ of the dispersive element is thus maintained constant. Adiabatic tapering Section II is identical for all the waveguides, so that it does not affect the demultiplexer dispersion.

The length difference $\Delta L^{\prime}$ of the arrayed waveguides is chosen such that the phase difference between adjacent waveguides equals an integer multiple of $2 \pi$ for the central wavelength $\lambda_{\mathrm{c}}$ of the dispersive element. For this $\lambda_{c}$, the signals in the individual waveguides will reach the output aperture with equal phase modulo $2 \pi$. The dispersion of the element is due to the linearly decreasing (increasing) lengths $L_{j}\left(L_{j}^{\prime}\right)$ of Sections I and III. The wavelength-dependent phase shift between adjacent waveguides produces a wavelength-dependent tilt angle of the wavefront in the output combiner, so that different wavelengths converge toward different points along an arc (Rowland circle) 


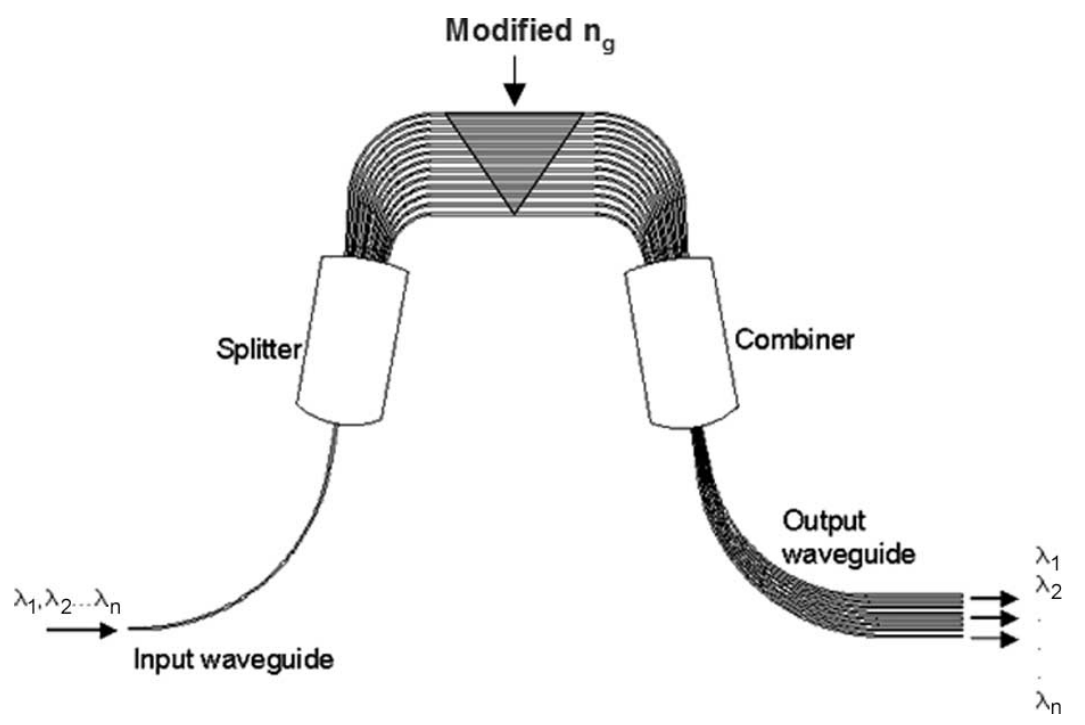

Fig. 2. Dispersion enhancement in a conventional AWG by group-index modification. The waveguide group index is modified in the indicated triangular-shaped region of the phased array.

of a radius $R / 2$ [19], [20], as it is shown in the receiver geometry in Fig. 1(c).

We denote the effective index in waveguide Sections I and III as $n(\lambda, W)$, with $W$ either $W_{0}$ or $W_{1}$. The phase shift $\Delta \phi$ between two adjacent waveguides is

$$
\Delta \phi\left(\lambda, W_{0}, W_{1}\right)=\frac{2 \pi}{\lambda}\left(n\left(\lambda, W_{1}\right)-n\left(\lambda, W_{0}\right)\right) \Delta L^{\prime} .
$$

By requiring that the phase shift between the adjacent waveguides is constant for all the waveguides and is equal to an integer multiple of $2 \pi$ for the central wavelength $\lambda_{c}$, we obtain

$$
\Delta L^{\prime}\left(\lambda_{\mathrm{c}}, m, W_{0}, W_{1}\right)=\frac{\lambda_{\mathrm{c}} m}{n\left(\lambda_{\mathrm{c}}, W_{1}\right)-n\left(\lambda_{\mathrm{c}}, W_{0}\right)}
$$

where $m$ is the order of the phase array.

Using (1) and (2) with angle dispersion relation (see, e.g., Smit and van Dam [3]) and the approximation $\sin (x) \sim x$, the wavefront tilt angle $\theta$ and the free spectral range (FSR) are

$$
\begin{aligned}
\theta\left(m, \lambda, W_{0}, W_{1}\right) & =\frac{M\left(m, \lambda_{\mathrm{c}}, W_{0}, W_{1}\right)}{n_{\mathrm{FPR}} d_{\mathrm{a}}} \Delta \lambda \\
\operatorname{FSR}\left(m, \lambda, W_{0}, W_{1}\right) & =\frac{\Delta \lambda}{M\left(m, \lambda_{\mathrm{c}}, W_{0}, W_{1}\right)}
\end{aligned}
$$

where $n_{\mathrm{FPR}}$ is the effective mode index in the free propagating slab-waveguide region, and $d_{\mathrm{a}}$ is the waveguide pitch where the array joins the output combiner [see Fig. 1(c)]. Here, we have considered the Taylor expansion to the first order in $\Delta \lambda=\lambda-\lambda_{c} . M$ in (3) and (4) is the modified grating order defined by

$$
M\left(\lambda_{\mathrm{c}}, m, W_{0}, W_{1}\right)=m \frac{n_{\mathrm{g}}\left(\lambda_{\mathrm{c}}, W_{1}\right)-n_{\mathrm{g}}\left(\lambda_{\mathrm{c}}, W_{0}\right)}{n\left(\lambda_{\mathrm{c}}, W_{1}\right)-n\left(\lambda_{\mathrm{c}}, W_{0}\right)}
$$

and $n_{\mathrm{g}}(\lambda, W)$ is the group index

$$
n_{\mathrm{g}}(\lambda, W)=n(\lambda, W)-\lambda \frac{d n(\lambda, W)}{d \lambda} .
$$

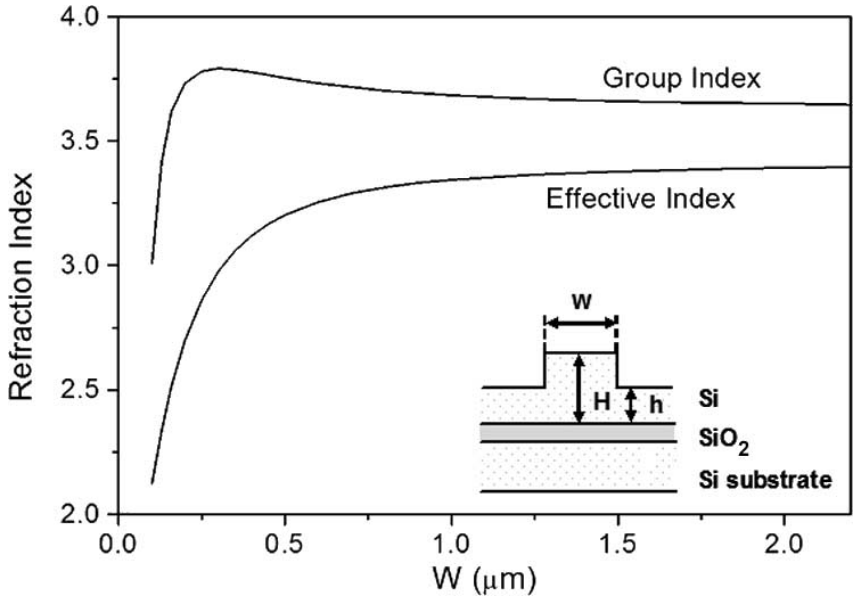

Fig. 3. Group index and effective index as a function of waveguide width. SOI ridge waveguide, etch depth $H-h=0.5 \mu \mathrm{m}$, SOI with a silicon layer of thickness $H=0.8 \mu \mathrm{m}$, and $\lambda_{\mathrm{c}}=1550 \mathrm{~nm}$.

The meaning of the modified interference order $M$ defined in (5) is similar to the modified grating order in a conventional AWG device [3], and it governs the device wavelengthdispersion characteristics. In addition to the parameters determining dispersion in a conventional AWG such as $(m$, $n_{\mathrm{FPR}}, d_{\mathrm{a}}$ ), here, an additional degree of freedom is provided by adjusting the group and effective index differences in (5). Fig. 3 shows the calculated dependence of the group index and the effective index on waveguide width for an example of SOI ridge waveguide etched $0.5 \mu \mathrm{m}$ deep in $0.8-\mu \mathrm{m}$-thick silicon layer.

From (5) and Fig. 3, it is observed that the (de)multiplexer dispersion can be controlled by adjusting the group index and the effective index values for different sections [Fig. 1(b), Sections I and III], for example, by a proper selection of the widths $W_{0}$ and $W_{1}$ of the staircase-like waveguides of the AWG. The modification of the interference order $M$, and hence, dispersion properties are a key finding of this paper. Equation (5) assumes the effect of waveguide-width modification 


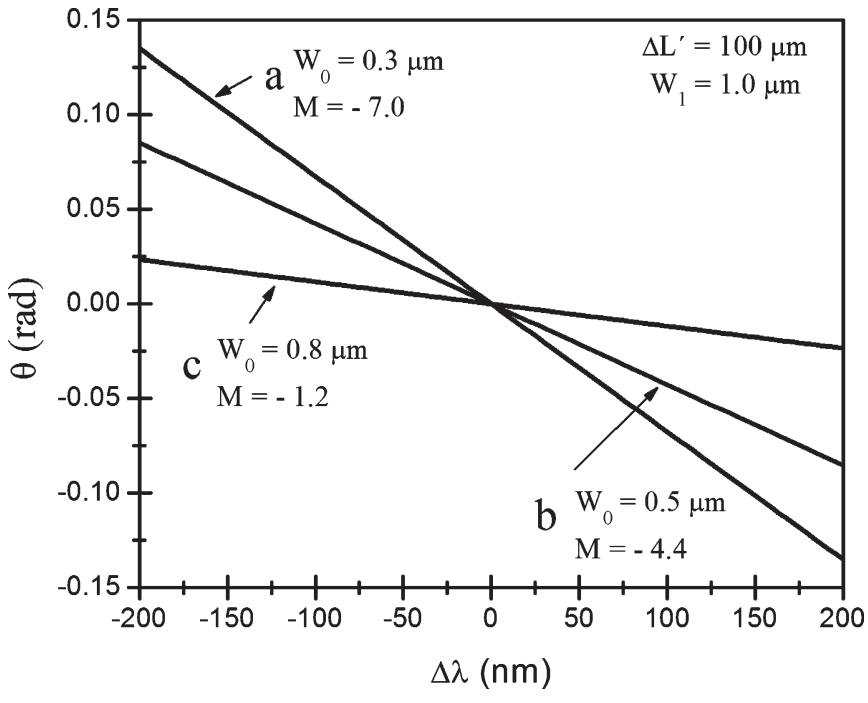

Fig. 4. Angular dispersion in SOI waveguides as a function of waveguide width $W_{0} . W_{1}=1.0 \mu \mathrm{m}$ is constant in the calculations. Calculated modified interference order $M$ is also shown.

according to the schematic shown in Fig. 1(b), but it can be generalized for any waveguide modification that results in a change of $\Delta n_{\mathrm{g}}$ in the group index and of $\Delta n$ in the effective index between the two waveguide sections

$$
M=m \frac{\Delta n_{\mathrm{g}}}{\Delta n} .
$$

The wavelength dispersion was calculated for a dispersive element designed for the SOI platform. We assume an array of $N=200 \mathrm{Si}$ ridge waveguides etched $0.5 \mu \mathrm{m}$ deep in $0.8 \mu \mathrm{m} \mathrm{Si}$ of refractive index $n_{\mathrm{Si}}=3.476$ at $1550 \mathrm{~nm}$, an $\mathrm{SiO}_{2}$ cladding of thickness of $1 \mu \mathrm{m}$, refractive index $n_{\mathrm{SiO}_{2}}=1.5$, waveguide pitch $d_{\mathrm{a}}=3 \mu \mathrm{m}$, effective index in slab waveguide $n_{\mathrm{FPR}}=$ 3.44 , and central wavelength $\lambda_{\mathrm{c}}=1550 \mathrm{~nm}$. The waveguide width is tapered to $2.5 \mu \mathrm{m}$ before joining the slab waveguides to reduce diffraction loss. The mode field distribution and propagation constants are calculated by a vectorial finite element method (FEM) mode solver.

The taper length [Fig. 1(b), Section II] was assumed to be $100 \mu \mathrm{m}$. Adiabatic gradual tapering between the waveguide sections of different widths assures that loss is minimized and no higher order modes are excited where the two waveguides join. We studied adiabatic SOI tapers theoretically and experimentally elsewhere [21], demonstrating that for taper length $>50 \mu \mathrm{m}$, both the loss penalty and the mode conversion are negligible; hence, the taper influence was not included in the simulation here.

Fig. 4 shows spectral dependence of dispersion angle $\theta$ in a first order approximation according to (3). Waveguide parameters have been chosen such that condition (2) is satisfied for a length difference $\Delta L^{\prime}$ of $100 \mu \mathrm{m}$, keeping the size of the dispersive-element constant.

An approximate estimate of the CS can be obtained using the Rayleigh criterion

$$
\mathrm{CS}\left(m, W_{0}, W_{1}\right)=\frac{1.22 \lambda_{\mathrm{c}}}{N M\left(m, \lambda_{\mathrm{c}}, W_{0}, W_{1}\right)} .
$$

CS dependence on $W_{0}$ is shown in Fig. 5 for a waveguide width of $W_{1}=1 \mu \mathrm{m}$. Square, circle, and triangle data points show the CS calculated for $\Delta L^{\prime}=50,100$, and $150 \mu \mathrm{m}$, respectively. CS of $1 \mathrm{~nm}$ or less is estimated for the device geometry showed in this example. From (8) and (4), we estimate approximate number of the output channels for a device designed for the SOI platform with the characteristics $N=200$, $\lambda_{\mathrm{c}}=1550 \mathrm{~nm}, d_{\mathrm{a}}=3 \mu \mathrm{m}, W_{0}=0.3 \mu \mathrm{m}, W_{1}=1.0 \mu \mathrm{m}$, $\Delta L^{\prime}=100 \mu \mathrm{m}$, hence $\mathrm{CS}=1.4 \mathrm{~nm}$ yielding 442 channels within FSR.

Fig. 6 shows the far field intensity of the dispersive element calculated by FEM. An array of $N=200$ waveguides was assumed. The waveguide width was tapered to $2.5 \mu \mathrm{m}$, where the arrayed waveguides join the output slab waveguides. A Gaussian intensity apodization was assumed across the phased array, as in a conventional AWG device. The calculated slit function (dashed curve envelope in Fig. 6) corresponding to the far-field diffraction by a single waveguide is also shown. A diffraction limited crosstalk is less than $-40 \mathrm{~dB}$. Detail of the farfield light distribution near $\theta=0$ is shown in Fig. 7. Calculated diffraction loss at the boundary between the waveguide array and the output slab waveguide is $-1.5 \mathrm{~dB}$.

Various geometries providing the imaging of the input waveguide onto the focal curve can be combined with the proposed dispersive element, including the zero-order AWG suggested by Adar et al. [22], schematically shown in Fig. 1(a). Discussion on such geometries is out of the scope of this paper.

It should be noted that the device based on the modification of waveguide width is intrinsicaly polarization dependent due to birefringence variation with waveguide cross-sections. For application where polarization insensitivity is demanded, this problem may be resolved for example by depositing a stressinducing cladding material such as stressed silicon dioxide layer with different thickness over the respective sections. In such case, the waveguide birefringence can be effectively eliminated by cladding-stress-induced elastooptical effect, as proposed by Xu et al. [23].

\section{Dispersion Enhancement IN AWG}

In the following example, we demonstrate how the proposed method of group modification can be used to enhance the dispersion property of an AWG demultiplexer. It can be shown [by following similar arguments to those leading to (3) and (5)] that by placing a triangularlike section of the waveguides with modified group index (see Fig. 2) in a phased array of a conventional AWG, the following relation for the dispersion angle in the output combiner slab waveguide is obtained:

$$
\theta \approx \frac{\Delta \lambda}{n_{\text {slab }} \Lambda}\left(M_{\text {conv }}+M\right)=\frac{\Delta \lambda}{n_{\text {slab }} \Lambda} \frac{n_{\mathrm{g}} \Delta L+\Delta n_{\mathrm{g}} \Delta L_{\mathrm{g}}}{\lambda_{\mathrm{c}}}
$$

where $M_{\text {conv }}=n_{\mathrm{g}} m / n_{\mathrm{eff}}$ is the order of a conventional AWG with length increment $\Delta L$ between the adjacent waveguides, $m=\Delta L n_{\text {eff }} / \lambda_{\mathrm{c}}, M$ is the interference order enhancement factor (7) due to group-index modification, and $\Delta L_{\mathrm{g}}$ is the length difference between the adjacent waveguides with modified group index. 


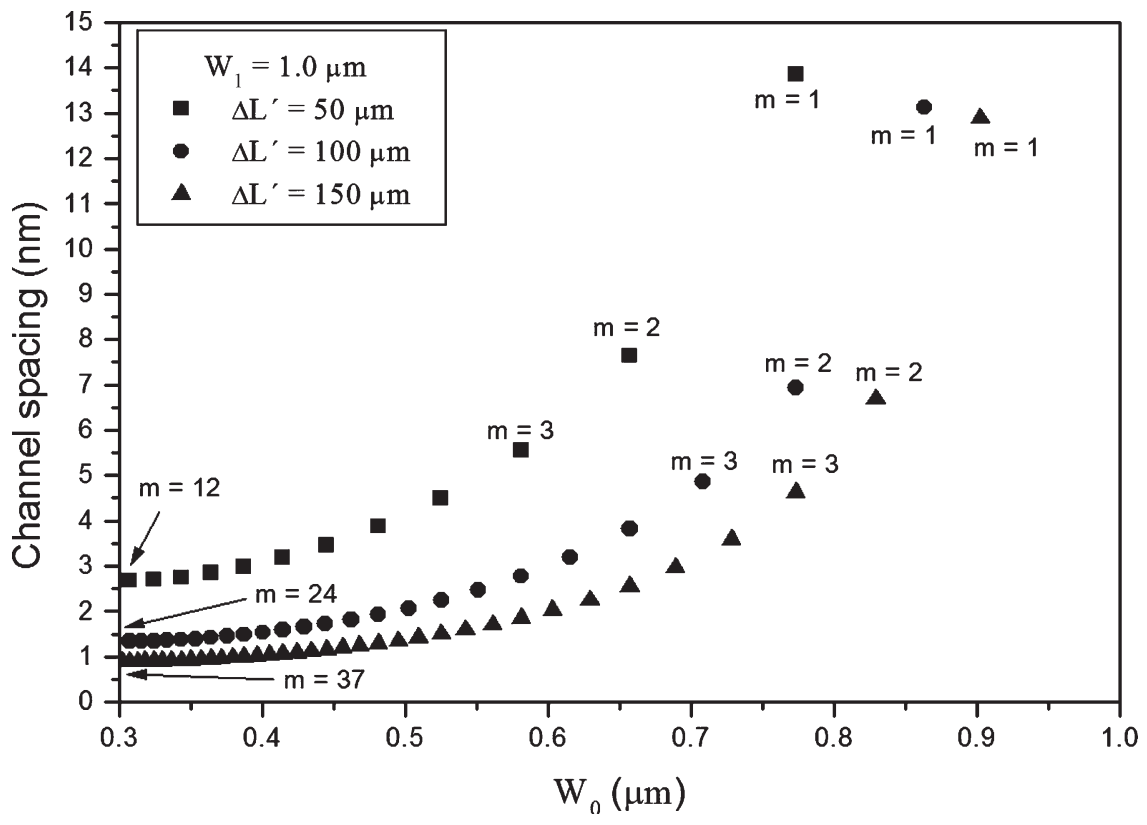

Fig. 5. CS as a function of waveguide width $W_{0}$ for different values of the length $\Delta L^{\prime}$ (see inset). Data points are calculated for $W_{1}=1 \mu \mathrm{m}$, and $m$ values associated to data points are shown.

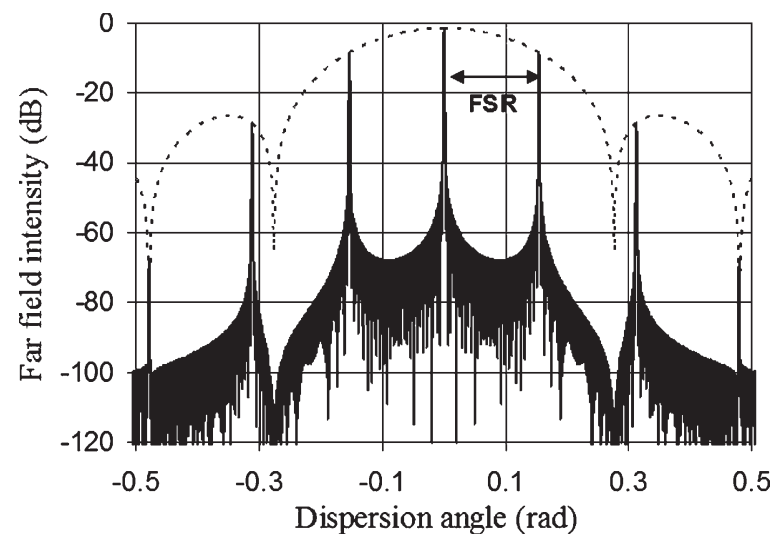

Fig. 6. Far field intensity of the dispersive element. Calculated by FEM for an array of $N=200$ SOI ridge waveguides with a pitch of $3 \mu \mathrm{m}$.

Now, we calculate the dispersion enhancement due to the element with a modified group index inserted in a nine-channel SOI AWG device. The waveguides in the array are ridge waveguides with a nominal width of $2 \mu \mathrm{m}$. The phased array comprises 100 waveguides with a linear length increment of $\Delta L=22.68 \mu \mathrm{m}$ with a grating order of $m=49$ and a modified order $M=50$, hence, with an FSR of $31 \mathrm{~nm}$. Minimum separation between the arrayed waveguides is $4 \mu \mathrm{m}$ at both ends of the phased array. The arrayed waveguide is tapered from a nominal width of 2 to $3 \mu \mathrm{m}$ at the ends of the array. The calculated linear dispersion is $0.044 \mu \mathrm{m} / \mathrm{GHz}$. The separation between the output waveguides at the output coupler focus located at the Rowland circle is $8.8 \mu \mathrm{m}$, and the focal length of the combiner (coupler) slab regions is $1.5 \mathrm{~mm}$. This design gives a Gaussian passband with a $200-\mathrm{GHz}$ CS and a calculated crosstalk of $<-30 \mathrm{~dB}$, a total diffraction loss of $-4 \mathrm{~dB}$, and a channel nonuniformity of $0.2 \mathrm{~dB}$. The same design yields an 18-channel device at $100-\mathrm{GHz}$ grid providing the separation between the output waveguides at the Rowland circle is reduced

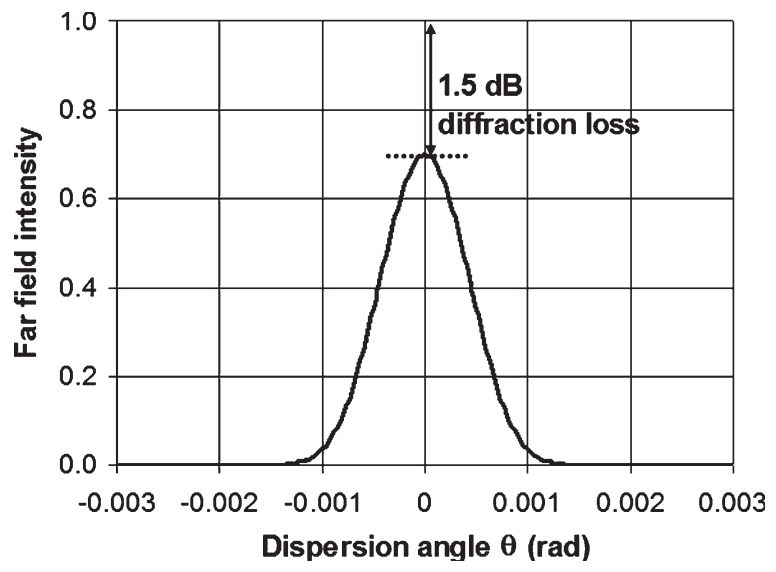

Fig. 7. Dispersive-element far field intensity distribution near $\theta=0$.

from 8.8 to $4.4 \mu \mathrm{m}$. Further design details and experimental results have been published elsewhere [24]. Upon inserting our dispersive element with a modified group index into the phased array of the AWG, the modified AWG dispersion can be estimated according to (9). It is particularly attractive to induce the group-index modification by inserting a waveguide section with a large group index. The latter can be obtained near the edge of the stopband of various structures, including gratings, resonators, or photonic crystals. Fig. 8 shows the dispersion enhancement calculated from (9) in the SOI AWG described above by using a dispersive element with a group-index modification as measured in photonic-bandgap SOI waveguides recently reported by Nippon Telegraph and Telephone (NTT) [25]. A nonlinear dependence of $n_{\mathrm{g}}(\lambda)$ (with $\Delta n_{\mathrm{g}} \sim 90$ near $1520 \mathrm{~nm}$ ), as in [25, Fig. 4(b)], was used for dispersion-enhancement estimate in (9). A large enhancement of modified order $M$ (compared to $m$ ), and hence of AWG angular dispersion, is observed in Fig. 8. The results shown here are meant to illustrate the potential of the proposed 


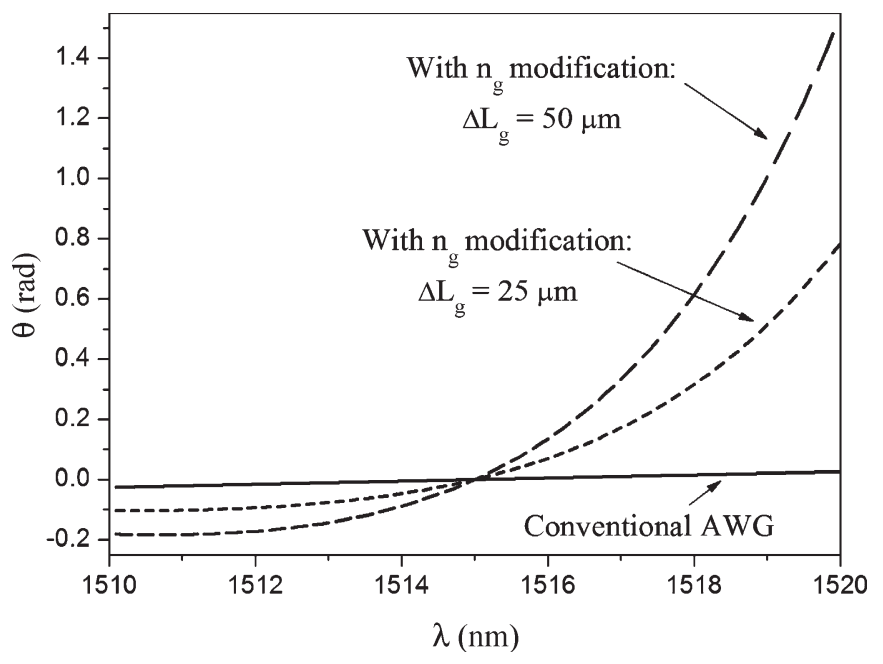

Fig. 8. Dispersion enhancement in an AWG device by waveguide group-index modification. Dispersive element with a modified group index by photonicbandgap effect is inserted into the phased array of a conventional AWG, as it is schematically shown in Fig. 2. Dispersion enhancement is calculated from (9) for $M_{\text {conv }}=50$ and for the length differences $\Delta L_{\mathrm{g}}$ between the adjacent waveguides with modified group index of 50 and $25 \mu \mathrm{m}$. Data for nonlinear dependence of $n_{\mathrm{g}}(\lambda)$ in (9) were taken from [25].

dispersion-enhancement mechanism. The future work will address the detailed device design and optimization, including the detailed simulation of photonic-bandgap waveguides and mode matching between the conventional and the photonicbandgap waveguides.

\section{SUMMARY}

We have proposed a new wavelength-dispersive principle based on the waveguide group-index modification and have shown how this principle is applied in a wavelength-dispersive element using a waveguide array with two sections of modified group index. An advantage of this element is its compactness as it does not require bent waveguides. Dispersive properties are estimated for an element implemented in an SOI platform. It is also shown how the proposed group-index modification can be used to markedly enhance dispersion of a conventional AWG. This is shown for an example of a conventional AWG designed in SOI platform using the proposed dispersion modification formula and the experimental data for groupindex modification in SOI photonic-bandgap waveguides. This dispersive element is also relevant for a recently reported interleaved FT Michelson-type AWG microspectrometer with large étendue [18].

\section{REFERENCES}

[1] J. Y. Wei, "Advances in the management and control of optical internet," IEEE J. Sel. Areas Commun., vol. 20, no. 4, pp. 768-785, May 2002.

[2] J. Zhang and B. Mukherjee, "A review of fault management in WDM mesh networks: Basic concepts and research challenges," IEEE Network, vol. 18, no. 2, pp. 41-48, Mar./Apr. 2004.

[3] M. K. Smit and C. van Dam, "PHASAR-based WDM devices: Principles, design, and application," IEEE J. Sel. Topics Quantum Electron., vol. 2, no. 2, pp. 236-250, Jun. 1996.
[4] S. Janz, A. Balakrishnan, S. Charbonneau, P. Cheben, M. Cloutier, A. Delâge, K. Dossou, L. Erickson, M. Gao, P. Krug, B. Lamontagne, M. Packirisamy, M. Pearson, and D.-X. Xu, "Planar waveguide echelle gratings in silica-on-silicon," IEEE Photon Technol. Lett., vol. 16, no. 2, pp. 503-505, Feb. 2004.

[5] J.-J. He, B. Lamontgne, A. Delage, L. Erickson, M. Davies, and E. S. Koteles, "Monolitic integrated wavelength demultiplexer based on a waveguide Rowland circle grating in InGaAsP/InP," J. Lightw. Technol., vol. 16, no. 4, pp. 631-638, Apr. 1998.

[6] V. I. Tolstikhin, A. Densmore, K. Pimenov, Y. Logvin, S. Laframboise, and S. Grabtchak, "Monolithically integrated optical channel monitor for DWDM transmission systems," J. Lightw. Technol., vol. 22, no. 1, pp. 146-153, Jan. 2004.

[7] S. Janz, M. Pearson, B. Lamontagne, L. Erickson, A. Delâge, P. Cheben, D.-X. Xu, M. Gao, A. Balakrishnan, J. Miller, and S. Charbonneau, "Planar waveguide echelle gratings: An embeddable diffractive elements for photonics integrated circuits," in Proc. Optical Fiber Communication Conf., OSA Tech. Dig., Anaheim, CA, 2002, vol. 70, pp. 69-70.

[8] R. R. Whiteman, A. P. Knights, D. George, I. E. Day, A. Vonsovici, A. A. House, G. F. Hopper, and M. Asghari, "Recent progress in the design, simulation and fabrication of small cross-section silicon-on-insulator VOAs," in Proc. SPIE, Photonocs West, San Jose, CA, 2003, vol. 4997, pp. $146-156$.

[9] L. Pavesi and D. J. Lockwood, Silicon Photonics. New York: SpringerVerlag, 2004.

[10] G. T. Reed and A. P. Knights, Silicon Photonics-An Introduction. Chichester, U.K.: Wiley, 2004.

[11] P. Cheben, D.-X. Xu, S. Janz, and A. Delâge, "Scaling down photonic waveguide devices on the SOI platform," in Proc. SPIE, J. F. Lopez, J. A. Montiel-Nelson, and D. Pavlidis, Eds, Maspalomas, Spain, 2003, vol. 5117, VLSI Circuits and Systems, pp. 147-156.

[12] V. R. Almeida and M. Lipson, "Optical bistability on a silicon chip," Opt. Lett., vol. 29, no. 20, pp. 2387-2389, Oct. 2004.

[13] M. R. T. Pearson, A. Bezinger, A. Delâge, J. W. Fraser, S. Janz, P. E. Jessop, and D.-X. Xu, "Arrayed waveguide grating demultiplexers in silicon-on-insulator," in Proc. SPIE, San Jose, CA, 2000, vol. 3953, pp. $11-18$.

[14] S. Janz, "Silicon-based waveguide technology for wavelength division multiplexing," in Silicon Photonics. New York: Springer-Verlag, 2004, ch. 10 .

[15] T. Fukazawa, F. Ohno, and T. Baba, "Very compact arrayed-waveguidegrating demultiplexer using Si photonic wire waveguides," Jpn. J. Appl. Phys., vol. 43, no. 5B, pp. L673-L675, May 2004.

[16] K. Sasaki, F. Ohno, and A. Motegi, "Arrayed waveguide grating of $70 \times 60 \mu \mathrm{m}^{2}$ size based on Si photonic wire waveguides," Electron. Lett., vol. 41, no. 14, pp. 801-802, Jul. 2005.

[17] Y. Kawakita, T. Saitoh, S. Shimotaya, and K. Shimomura, "A novel straight waveguide grating with linearly varying refractive-index distribution," IEEE Photon. Technol. Lett., vol. 16, no. 1, pp. 144-146, Jan. 2004.

[18] P. Cheben, I. Powell, S. Janz, and D.-X. Xu, "Wavelength-dispersive device based on a Fourier-transform Michelson-type arrayed waveguide grating," Opt. Lett., vol. 30, no. 14, pp. 1824-1826, Jul. 2005.

[19] H. A. Rowland, "Preliminary notice of the results accomplished in the manufacture and theory of gratings for optical purposes," Phil. Mag., vol. 13, pp. 469-474, 1882 .

[20] - The Physical Papers of Henri August Rowland. Baltimore, MD: The Johns Hopkins Univ. Press, 1902, pp. 487-491.

[21] P. Cheben, A. Bogdanov, A. Delâge, S. Janz, B. Lamontagne, M. J. Picard, E. Post, and D.-X. Xu, "A 100-channel near-infrared SOI waveguide microspectrometer: Design and fabrication challenges," in Proc. SPIE, Beijing, China, 2005, vol. 5644, pp. 103-110.

[22] R. Adar, C. H. Henry, C. Dragone, R. C. Kistler, and M. A. Mildbrodt, "Broad-band array multiplexers made with silica waveguides on silicon," J. Lightw. Technol., vol. 11, no. 2, pp. 212-219, Feb. 1993.

[23] D.-X. Xu, P. Cheben, D. Dalacu, A. Delâge, S. Janz, B. Lamontagne, M.-J. Picard, and W. N. Ye, "Eliminating the birefringence in silicon-oninsulator ridge waveguides by use of cladding stress," Opt. Lett., vol. 29, no. 20, pp. 2384-2386, Oct. 2004.

[24] P. Cheben, D.-X. Xu, S. Janz, A. Delâge, and D. Dalacu, "Birefringence compensation in silicon-on-insulator planar waveguide demultiplexers using a buried oxide layer," in Proc. SPIE, San Jose, CA, 2003, vol. 4997, pp. 181-189.

[25] M. Notomi, K. Yamada, A. Shinya, J. Takahashi, C. Takahashi, and I. Yokohama, "Extremely large group-velocity dispersion of line-defect waveguides in photonic crystal slab," Phys. Rev. Lett., vol. 87, no. 25, pp. 253902-1-253902-4, Dec. 2001. 


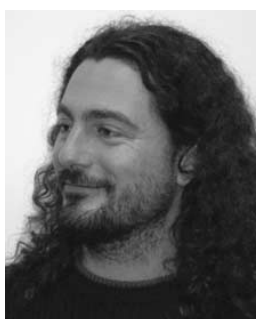

Óscar M. Matos was born in Orense, Spain, in September 7, 1972. He received the M.S. degree Madrid, Madrid, Spain, and the Ph.D. degree in physics from La Plata National University, La Plata, Argentina, in 1996 and 2002, respectively.

He worked in the diverse fields of physics, such as nonlinear optics (second harmonic generation, noncollinear phase matching, photorefractive effect), defects in solids, photoacoustic, photonic-waveguide devices, and holography. His current research interest is in holographic recording materials and arrayed waveguide grating devices. in physics science from Complutense University of

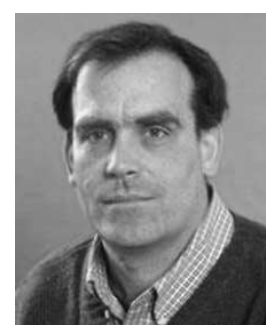

Siegfried Janz (M'98) received the Ph.D. degree in physics from the University of Toronto, Toronto, ON, Canada, in 1991, where his graduate research was in surface nonlinear optics.

After completing the degree, he joined the NRC of Canada, Ottawa, ON, where he worked on nonlinear frequency conversion and optical switching in III-V semiconductor waveguides and silicon-based optoelectronics. From 2001 to 2002, he was part of the research team at Optenia Inc., which successfully developed the first glass waveguide echelle grating demultiplexer. He is presently the Group Leader of the Optoelectronic Devices Group at the Institute for Microstructural Sciences, NRC, and also an Adjunct Professor with the Carleton University, Department of Electronics, Ottawa. His current research interests include active and passive integrated optics and, in particular, microphotonic devices in high-index contrast waveguide systems.

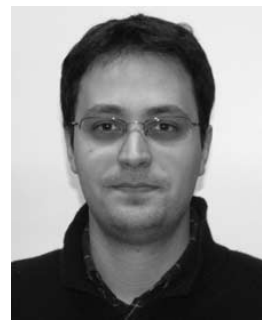

José A. Rodrigo was born in Madrid, Spain, in 1979. He received the M.S. degree in science from the Complutense University of Madrid, in 2003. He is currently working toward the Ph.D. degree in optical computing, optical signal processing, holography, and fractal optics at Complutense University.

Complutense University of Madrid, Madrid, Spain, in 1977.

From 1969 to 1971, she initiated research at the Institute of Optics, French National Center for Scientific Research (CNRS), on optical properties of glasses and amorphous materials. In 1972, she joined the Optics Department of Complutense University as an Assistant Professor. In 1981, she became an Associate Professor and, in 1999, Chair of Optics at the same department. She was a Visiting Professor at the University of California School of Optometry, Berkeley, in 1985-1986, 1988, and 1989. In 1993, she organized the Interdisciplinary Group for Bio-optics Research (GIBO-UCM), currently designated as Interdisciplinary Group for Optical Computing (GICO-UCM). She was also a Visiting Professor at the University of Missouri School of Optometry, St. Louis, in 1994 and 2000. She is the author of more than 100 scientific publications in Electromagnetic Theory of Optical Waveguides, Optical Signal Processing, and Holography and Bio-optics.

Dr. Padilla was elected Vice-President at the ICO Bureau (term 1999-2002) and is currently a Secretary General (term 2002-2005 and 2005-2008). She is a Fellow of the Optical Society of America (OSA) and a member of The International Society for Optical Engineers (SPIE), European Optical Society (EOS), and the Sociedad Española de Optica (SEDO).

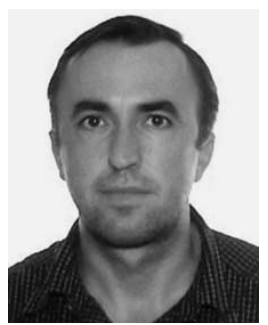

Pavel Cheben received the M.Sc. degree in optoelectronics from the Slovak Technical University, Bratislava, Slovakia, in 1990 and the Ph.D. degree in physics (optics) from the Complutense University of Madrid, Madrid, Spain, in 1997.

$\mathrm{He}$ is a Research Officer at the Institute for Microstructural Sciences at the National Research Council (NRC) of Canada, Ottawa, ON. He is also an Honorary Professor at the Department of Physics, Complutense University of Madrid. His research interests include integrated optics, silicon photonics, and optical materials. He has coauthored over 80 publications and research reports, three book chapters, and over 20 patent applications in integrated optics, photonics, and optical and photonic materials. He was a member of the team which started up Optenia, Inc., and developed the first commercial waveguide echelle grating wavelength division multiplexed (WDM) demultiplexer. Before joining the NRC, he was in charge of the foundation of the Laboratory of Space Instrumentation at the National Institute for Aerospace Technology of Spanish Ministry of Defense.

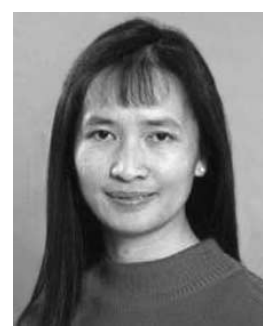

Dan-Xia Xu received the Ph.D. degree from Linköping University, Linköping, Sweden, in 1991 where she worked on silicon-germanium electronic components such as heterojunction bipolar transistors (HBTs).

From 2001 to 2002, she was part of the research team at Optenia Inc., which successfully developed the first glass waveguide echelle grating demultiplexer. She is a Research Officer at the NRC, Ottawa, ON, Canada, and also an Adjunct Professor at the Department of Electronics, Carleton University, Ottawa. Her research areas covered the material research, design and fabrication of optoelectronic and microelectronic components based on silicon, silicon on insulator (SOI), silicon-germanium alloys, and silicides. Her current research focuses on high-index contrast waveguide systems, particularly passive and active integrated optics based on the SOI platform and their applications in communications and biophotonics.

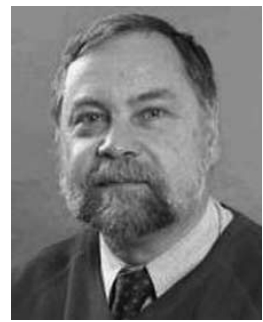

André Delâge was born in Quebec City, OC, Canada, on February 9, 1949. He received the B.A., B.Sc., M.Sc., and Ph.D. degrees in 1968, 1971, 1973, and 1976, respectively, all in physics from Laval University, Quebec City, where he was involved in electron spectrometry.

After a year at the Institut National des Sciences Appliquées, Lyon, France, where he worked in surface physics and a postdoctoral fellowship with Bomem in infrared Fourier transform (FT) spectrometry, he joined the Electron Physics Section, Division of Electrical Engineering, at the NRC, Ottawa, ON, Canada, in 1978 to work on the development of the lanthanum hexaboride source for the scanning electron microscope. During this period, he developed an expertise in numerical analysis associated with electron optics projects. After his group joined the new Institute of Microstructural Sciences in 1987, he was transferred to the Optoelectronics group, where he is currently responsible for the modeling of waveguide optics and optoelectronic devices. From 2001 to 2002, he led the numerical simulation group at Optenia to commercialize the WDM technology developed at NRC. Subsequently, he returned to NRC to continue his activities in microphotonics. 